\title{
Review
}

Franziska Ullm and Tilo Pompe*

\section{Fibrillar biopolymer-based scaffolds to study macrophage-fibroblast crosstalk in wound repair}

https://doi.org/10.1515/hsz-2021-0164

Received February 24, 2021; accepted July 9, 2021; published online August 16, 2021

\begin{abstract}
Controlled wound healing requires a temporal and spatial coordination of cellular activities within the surrounding extracellular matrix (ECM). Disruption of cellcell and cell-matrix communication results in defective repair, like chronic or fibrotic wounds. Activities of macrophages and fibroblasts crucially contribute to the fate of closing wounds. To investigate the influence of the ECM as an active part controlling cellular behavior, coculture models based on fibrillar 3D biopolymers such as collagen have already been successfully used. With well-defined biochemical and biophysical properties such 3D scaffolds enable in vitro studies on cellular processes including infiltration and differentiation in an in vivo like microenvironment. Further, paracrine and autocrine signaling as well as modulation of soluble mediator transport inside the ECM can be modeled using fibrillar 3D scaffolds. Herein, we review the usage of these scaffolds in in vitro coculture models allowing in-depth studies on the crosstalk between macrophages and fibroblasts during different stages of cutaneous wound healing. A more accurate mimicry of the various processes of cellular crosstalk at the different stages of wound healing will contribute to a better understanding of the impact of biochemical and biophysical environmental parameters and help to develop further strategies against diseases such as fibrosis.
\end{abstract}

Keywords: coculture; collagen I; 3D scaffolds; immune cells; wound healing.

\footnotetext{
*Corresponding author: Tilo Pompe, Faculty of Life Sciences, Institute of Biochemistry, Leipzig University, D-04103 Leipzig, Germany, E-mail: tilo.pompe@uni-leipzig.de. https://orcid.org/0000-00031508-4959

Franziska Ullm, Faculty of Life Sciences, Institute of Biochemistry, Leipzig University, D-04103 Leipzig, Germany. https://orcid.org/ 0000-0002-0547-7540
}

\section{Introduction}

Tissue homeostasis is a well-orchestrated process that requires perpetual communication between cells and their surrounding extracellular matrix (ECM). In case of an injury, repair processes are immediately initiated to secure and/or restore the disturbed tissue, activating wound healing processes. This multi-stage process involves highly dynamic interactions between various cell types and tremendous cellular remodeling of the ECM, involving dermal and epidermal cells, macrophages (MФ), fibroblasts (FB), and endothelial cells in cutaneous wound healing. Failure to induce, terminate or regulate a certain stage leads to pathological wound healing and the loss of tissue function (Eming et al. 2014).

Such uncontrolled wound healing like fibrosis or chronic inflammation are frequently found in patients with multi-disease states, which are increasing incidence in the modern societies (Eming et al. 2017; Sindrilaru and Scharffetter-Kochanek 2013). Although physiological wound healing is usually well controlled in the clinics, pathological wound healing situations ask for a better understanding of the various stages and cellular interactions during wound healing and approved therapies. In vivo studies on animal models are frequently used in this context, but are ethically controversial and the scientific results are also questioned, as the results of studies on "healthy animals" are transferred to therapies on "multidiseased humans" and successful translation often fails.

On the other side standard two-dimensional (2D) in vitro cell culture studies were questioned during the last decades. Many cell biological processes are strongly dependent on the microenvironment including adhesive, micro- and nano-structural, and mechanical characteristics of cell culture scaffolds as well as the presentation of soluble and bound mediators. Hence, three-dimensional (3D) models have been developed as improved in vitro cell culture scaffolds to better mimic these decisive parameters in physiological and pathological situations (Baker and Chen 2012; Charras and Sahai 2014; Chaudhuri et al. 2020; Doyle and Yamada 2016; Freudenberg et al. 2016; Sapudom 
and Pompe 2018). In this field, 3D matrices based on natural biopolymers such as collagen I have been evolved as a highly relevant scaffold material especially when focusing on cutaneous wound healing (Place et al. 2009), because collagen I is very abundant in the native ECM and can mimic various important ECM features in vitro, like fibrillar 3D network structure, ligand specificity, and tuneability of network structure and mechanics.

In this review, we provide an overview on biomimetic in vitro 3D models to study cellular interactions between different cell types in wound healing. Thereby, we focus on the highly relevant models of fibrillar 3D cell culture scaffolds applied in studies on different stages of cutaneous wound healing, in particular on the interplay of МФ and FB as central cell types in regulating wound repair and immune response.

\section{Instructive 3D biomaterial scaffolds to study cutaneous wound healing in vitro}

Every tissue in the human body has its own composition of ECM proteins and cells resulting in an unique architectural design (Bonnans et al. 2014). The challenge in current engineering biomimetic cell culture scaffolds is to find appropriate materials exhibiting the defined mechanical, biochemical and structural characteristics of the specific tissue. Meanwhile, it is well known that cells growing on stiff 2D tissue culture plastics usually show a different behavior than cells growing in 3D structures (Caliari and Burdick 2016). Many patho-/physiological processes exclusively take place in a 3D context, such as gastrulation, spreading of blood vessels, and cancer cell migration during metastasis (Baker and Chen 2012). It is well accepted that a broad range of (bio)material parameters is essential to mimic the in vivo patho-/physiological ECM situation and that these parameters act in a highly convoluted manner on cell behavior (Chantre et al. 2019). Such parameters predominantly but not exclusively include adhesion ligands, nano- and microstructure, viscoelastic mechanics, structural dynamics, presentation of soluble mediators and cytokines and modulation of their activity, and degradability and hydration state (Bonnans et al. 2014; Chantre et al. 2019; Prewitz et al. 2012).

Hence, these scaffolds should provide mechanical stability to be self-supportive and provide structural support of cells, enable the exchange of nutrients and residues by sufficient porosity/mesh size, allow for cell adhesion via specific cell-binding motifs like RGD peptides or proteins i.e. collagen, fibronectin or laminin, and facilitate specific biological activity by addition of soluble or insoluble factors (Baker and Chen 2012; Baker et al. 2015). Modulation of these material parameters controls various cellular processes such as cell migration and contractility, cell-cell signaling and contact formation, matrix remodeling, formation of multicellular interactions, and downstream processes like proliferation, differentiation and apoptosis.

A successful engineering of such instructive biomaterial scaffolds to achieve a desired cell response have been based on various approaches, which can be basically classified into synthetic vs. natural materials in terms of material's origin. Dense vs. porous or fibrillar materials with respect to the 3D structure at the cell size level is another important fundamental classification scheme, crucially controlling multiple cellular communication pathways. Additionally, polymeric materials dominate the field due to the associated fundamental characteristics of the ECM, which is of biopolymeric origin. The mentioned classifiers can be considered as a good general scheme in biomaterial engineering of cell culture scaffolds. They summarize key features of engineered 3D scaffolds to study cell-cell communication as focused in this review. The choice of synthetic $v s$. natural materials has to balance the precision of scaffold construction and reproducibility $v s$. the ease to mimic biological ligand features. On the other side, pore size is particularly relevant with respect to the use of dense hydrogels with nanometer mesh size compared to fibrillar/porous scaffolds with micrometer pore size. It tightly controls juxtacrine and paracrine cell-cell communication, like the exchange of soluble signals as well as cell migration and direct cell-cell contact.

Scaffolds of synthetic origin are available as multifunctional hydrogel networks as well as stiff bulk materials, and porous or fibrillar scaffolds, depending on the synthesis and production scheme. Many approaches and polymer types exist, while widely known and applied polymers include poly(lactic)- or poly(glycolic) acid (PLA, PGA, respectively) and polyethylene glycol (PEG). Early on PEG-based hydrogels were shown as versatile synthetic polymers to mimic natural ECMs in terms of various biochemical and biophysical properties. Various protocols exist to fabricate hydrogels with tunable stiffness, coupling proteins and cell-binding ligands, as well as cell compatible cross-linking mechanisms and adjustable degradability (Infanger et al. 2013; Lutolf and Hubbell 2005). Furthermore, PEG-based scaffolds exhibit low unspecific protein adsorption. Early examples showed a good viability of encapsulated cells with efficient ECM deposition during degradation (Bryant and Anseth 2002), 
incorporation of cell adhesion ligands to the otherwise non-adhesive gels (Lutolf et al. 2003), or the introduction of bifunctional peptide linkers with enzymatically cleavable and cell-adhesive sequences to control 3D growth and matrix remodeling by endothelial cells (Tsurkan et al. 2010). In recent years, glycopolymers, particularly glycosaminoglycans (GAG), have also been incorporated into PEG-hydrogels, providing new options for cytokine binding, presentation, release, and sequestering (Freudenberg et al. 2016; Lohmann et al. 2017).

As outlined above, the 3D polymeric characteristics of biomimetic scaffolds were found to be essential for wound healing studies. Naturally occurring biopolymer materials like hyaluronan, chitosan or alginate were used as interesting and broadly applied options, too (Caliari and Burdick 2016; Haldar et al. 2019; Ter Horst et al. 2018). However, these dense polymer-based 3D hydrogels cannot resemble important features of the cutaneous ECM in wound healing being micron-sized pores of fibrillar networks and other important features of the skin physiology (Achterberg et al. 2014; Metcalfe and Ferguson 2007). Furthermore, degrading synthetic materials were frequently discussed to inhibit wound healing process, in particular by prolonging the inflammatory foreign body response (Groeber et al. 2011; van der Veen et al. 2010).

As alternative approaches, fibrillar 3D scaffolds based on natural biopolymers are widely investigated and used in biomaterials engineering, such as scaffolds based on fibrin and collagen. 3D fibrin networks, as mimics of the initial wound closure matrix are widely used in the clinics for straightforward wound closure applications. However, its versatile usage for defined biomimetic 3D scaffolds in long term cell-culture studies is hampered by the limited knowledge on precise engineering of defined network structures with targeted modifications and a low long-term stability against enzymatic degradation in cell culture (Rowley et al. 2019; Whelan et al. 2014).

In contrast, collagen I based 3D scaffolds have been shown to provide highly relevant properties because collagen I is highly abundant as ECM protein in skin tissue, and reconstituted networks exhibit a nano- and microstructure and multifunctional characteristics closely related to its in vivo ECM occurrence. In vitro reconstituted 3D fibrillar collagen I networks exhibit native adhesion cell receptor binding sites, biomimetic viscoelastic, and tunable mechanical properties, degradability by matrix metalloproteases (MMP), and 3D migration and invasion of various cell types (Doyle and Yamada 2016; Lang et al. 2015; Licup et al. 2015; Rahmany and van Dyke 2013; Rohani and Parks 2015; Sapudom et al. 2015b, 2019, 2020; Seo et al. 2020). Additionally, 3D collagen I networks can be functionally modified in terms of coupling other ECM components like glycoproteins and GAG (Kalbitzer et al. 2015; Sapudom et al. 2015b; Stamov et al. 2011; van der Smissen et al. 2013). Using such 3D collagen I scaffolds various wound healing situations have been mimicked in multiple studies e.g. using different human cell types like FB and keratinocytes (Ansorge et al. 2017; Iyer et al. 2018; Sapudom et al. 2015b, 2017c). As such, collagen I is a wellstudied 3D scaffold system, frequently functioning as a kind of gold standard in wound healing studies.

It remains to be noted that other interesting approaches in the design of instructive 3D biomaterial scaffolds to study cutaneous wound healing in vitro exist as well (Chouhan et al. 2019). Those scaffolds include the use of decellularized matrices with their advantages concerning highly biomimetic nano-/microstructure, composition and mechanics with concomitant disadvantages like high variety between charges and problems in immunogenicity (Rowley et al. 2019; Sadtler et al. 2017). Furthermore, 3D nanofibrillar scaffolds from synthetic self-assembling polypeptides (Caliari and Burdick 2016), multifunctional materials with hierarchical microstructure as well multi-parametric models (Jannasch et al. 2017; Xue et al. 2019), and advanced 3D (bio) printing technologies comprise recent developments with interesting material properties.

\section{On the importance of macrophages and fibroblasts - candidates to modulate wound healing}

Activated wound repair is a highly regulated cascade of enzymatic and cellular reactions typically divided into four stages: hemostasis, inflammation, proliferation and remodeling. As initial response, also called hemostasis, a provisional fibrin- and fibronectin-rich ECM is established within the blood coagulation cascade which stabilizes the site of injury and serves as a scaffold for invading cells presenting growth factors, e.g. TGF- $\beta$, PDGF, VEGF and FGF (Barker and Engler 2017). This provisional matrix further matures into a matrix predominantly containing fibronectin and proteoglycans, providing a template for subsequent collagen deposition (Sottile and Hocking 2002). This step triggers neutrophils, mast cells, and $M \Phi$ to migrate to the wound to remove cell debris, invaded microorganisms, pathogens, and degraded ECM components, a process also called inflammation (Singer and Clark 1999; Werner and Grose 2003). During inflammation, present cell types also function as important source of cytokines and metabolites, which leads to activation of keratinocytes and 
$\mathrm{FB}$, as well as their migration to and proliferation at the wound site. FB begin to replace the provisional ECM with fibrillar, collagen-rich ECM with higher mechanical strength (Tomasek et al. 2002). Some of the FB differentiate into myofibroblasts (MyoFB), a cell type contracting the wound to support closure and producing new ECM, mainly collagen type I and III (Gurtner et al. 2008). Part of the immune cells, like $M \Phi$, remain involved in processes securing the homeostasis of the tissue, including re-epithelialization and reorganization of the ECM (Rodero and Khosrotehrani 2010). Further alterations of the ECM structure occur in the last phase of wound healing, the remodeling phase. Highly dynamic interactions between dermal and epidermal cells, МФ, and endothelial cells occur that restore tissue integrity. Coordinated by growth factors and cytokines, most of the processes initiated during inflammatory phase, shut down and cease. Cells from earlier stages undergo controlled apoptosis or vanish from the wound site, leaving behind a matrix consisting of a few cells and mostly collagen I (Diegelmann and Evans 2004; Harding et al. 2002). Failure to induce, terminate or regulate a certain stage of wound healing leads to pathological wounds and loss of tissue function. Abnormal repair is mainly attributed to the prolonged presence of FB-activating $M \Phi$ and MyoFB driving inflammation and excessive remodeling of the ECM, which results in stiffening of the scar ECM and an uncontrolled accumulation of fibrotic tissue (Eming et al. 2017; Sindrilaru and ScharffetterKochanek 2013). Thus, the dynamic interactions between $\mathrm{M} \Phi$ and FB have proven as main regulators of fibrosis (Barron and Wynn 2011).

MФ are either derived from circulating blood monocytes or resides in the tissue being a part of the immune system. They release a broad range of cytokines and have multiple functions during tissue homeostasis but also orchestrate the restoration of tissue integrity and repair processes after injury. During all phases of wound healing, MФ fulfill essential roles. In a number of reviews, many authors have addressed the key function of MФ in mediating the immune response and the stages of tissue repair (Kloc et al. 2019; Koh and DiPietro 2011; Martin and García 2021; Minutti et al. 2017; Rodero and Khosrotehrani 2010; Snyder et al. 2016; Wynn and Vannella 2016; Wynn et al. 2013; Yanez et al. 2017). Of particular relevance is the fact that $\mathrm{M} \Phi$ can be activated in different ways, resulting in different subtypes with specific secretion profiles and functions and interaction pathways with the surrounding matrix and cells within wound healing, which was discussed in-depth in a recent review (Martin and García 2021). In vitro, $\mathrm{M} \Phi$ were initially classified into the $\mathrm{M} 1$ and $\mathrm{M} 2$ type, with fully polarized M1 and M2 cells rather representing the extremes of a continuum of subtypes (Mantovani et al. 2004). There is growing evidence that the type of stimulus or combination of stimuli controls the specific $\mathrm{M \Phi}$ response. M1 or classically activated macrophages are generated in response to the cytokine IFN- $y$ in combination with TNF or TNF-induced stimuli. Typically, those macrophages are prototypical immune effector cells which express high levels of inflammatory cytokines, reactive oxygen (ROS) and nitrogen species (RNS) to kill intracellular pathogens (Edwards et al. 2006; Nathan and Shiloh 2000). M1 macrophages are an important component of the host defense and a mediator of inflammation. M2 or alternatively activated macrophages were originally described as macrophages activated by IL-4, as opposed to classical activation by IFNy and LPS. They promote tissue remodeling and tumor progression, have antiparasitic effects and possess immunoregulatory properties. Now they are better divided into several variants. The M2a type is defined as activated by stimulation with IL-4 and IL-13. The M2b-type is related to an induction by TLR4 or IL-1R and immune complexes recognized by Fc-gamma receptors and the M2c-type is mainly induced by IL-10 or TGF- $\beta$ (Martinez et al. 2008). Due to their different functions during host response, wound healing and immune regulation, there are many activation modes and a broad spectrum of $М \Phi$ populations, with an ongoing discussion on classification markers and functions (Logie et al. 2020; Patel et al. 2017; Saeed et al. 2014). Also in the field of biomaterials and tissue engineering, numerous studies have addressed the prediction and control of the activation and polarization of certain MФ subtypes by the application of certain biomaterials (Jannasch et al. 2017; Witherel et al. 2016). It was found, that material properties can determine the МФ phenotype and are capable to generate mixed or completely new phenotypes (Graney et al. 2018). Hence it is concluded that a better understanding of the mechanisms to control $\mathrm{M} \Phi$ phenotype can support biomedical approaches in pathological wound healing.

FB and their differentiated phenotype, MyoFB strongly contribute to wound healing, too. Derived from different progenitors, MyoFB comprise a heterogeneous cell population with similar phenotype and function (Hinz 2007). Hallmarks of a MyoFB are the formation of prominent actin stress fibers, the ability to exert strong contractile forces and a high collagen production. The incorporation of alpha smooth muscle actin ( $\alpha S M A$ ) into stress fibers is widely used as phenotypic marker (Hinz 2016). Many studies have focused on fibrotic conditions, where MyoFB strongly contribute by their massive ECM production. To date, it is still unclear which mechanisms contribute early on to the development of fibrosis, as it is not possible to clearly 
differentiate between anti- and profibrotic behavior of MyoFB (Pakshir and Hinz 2018). Furthermore, specific markers are lacking which allow a timely diagnosis of early fibrosis. Numerous studies point to a disturbed communication between MФ and MyoFB as a cause for the development of fibrosis, since coordinated activities of МФ and MyoFB support physiological wound healing with downregulated MyoFB function (Ploeger et al. 2013; Shook et al. 2018; van Linthout et al. 2014; Venter and Niesler 2018).

With the strongly regulated and interactive behavior of MФ and FB in mind, it is obvious that studies on МФ-FB crosstalk are highly relevant to better understand the various processes in wound healing. In vivo data from Oka et al. (2016) from rats show the need for direct cell-cell contacts between $\mathrm{M} \Phi$ and FB in the maintenance of skin tissue structure and during wound healing (Oka et al. 2016). Another in vivo study in mice identified several subsets of wound bed MyoFB acting during skin repair (Shook et al. 2018). Distinct differences in proliferation and transcriptional heterogeneity were correlated to changes in aging and fibrosis. Additionally, $\mathrm{CD} 301 \mathrm{~b}^{+} \mathrm{M} \Phi$ were shown to selectively support the proliferation of adipocyte precursor cells, the most abundant MyoFB subset These results clearly show, that the wound bed ECM selectively activates specific subsets of FB and $М \Phi$ with implications on their crosstalk during skin repair. Indications of how the microenvironment determines cell plasticity during wound healing has also been obtained from in vitro studies. Secreted paracrine factors from different $М \Phi$ subtypes led to different FB phenotypes (Ploeger et al. 2013). Factors of M1-MФ produced a proinflammatory, ECM-degrading phenotype whereas factors secreted by M2-MФ induced proliferating $\mathrm{FB}$ and could even completely reverse the proinflammatory, ECM-degrading FB phenotype. Furthermore, coculture studies of human dermal FB and human monocyte-derived $\mathrm{M} \Phi$ revealed an inverse crosstalk with FB being inducers of a $M \Phi$ phenotype switch from inflammatory to an alternative/pro-repair type, mediated by the production of TSG6 and COX2 products (Ferrer et al. 2017).

\section{Biomimetic 3D in vitro models on macrophage-fibroblast crosstalk in wound healing}

As outlined in the previous chapters, there is a need to develop models for studies on the complex cell-cell interaction in cutaneous wound healing. $\mathrm{M} \Phi$ and FB were discussed for their extraordinary function at different stages of wound healing, although the influence of other cell types including neutrophils, epithelial cells, keratinocytes, and endothelial cells also have to be considered in order to reflect the complexity of the cellular interplay. Furthermore, it was addressed that biomimetic 3D scaffolds with well-defined material properties (microstructure, mechanics, degradability) have to be engineered allowing for cell-cell signaling and dynamics including juxtacrine, paracrine and autocrine signals as well as cellular migration, proliferation and differentiation. Such scaffolds should also enable high-resolution analytical techniques to investigate the spatiotemporal dynamics and heterogeneity of the complex cell system. Based on these considerations most approaches to study $\mathrm{M} \Phi$ and FB can be divided into models using cocultures of cell lines or primary cells, frequently from animals, and models with defined mimics of cellular signals of the corresponding cell type. A schematic overview of the crosstalk between $M \Phi$ and FB during different wound healing stages is depicted in Figure 1.

Early approaches to study the communication between MФ and FB were recently reviewed by Zhou and Groth (2018). They include studies applying conditioned media to a homotypic cell layer cultivated on planar or microstructured 2D surfaces, mostly to study biomaterial properties with respect to ECM modulating capabilities (Damanik et al. 2014; Fernando et al. 2016; Ploeger et al. 2013). Such approaches are restricted in terms of direct and continuous exchange of soluble mediators. Another method to study cells in coculture are separated membrane (transwell) systems, that prevent direct, heterotypic cell contacts but enable the exchange of soluble signals. Such a system with primary and secondary $\mathrm{M} \Phi$ and FB cell cultures and conditioned medium and a comparison of non-contact and contact coculture (paracrine $v s$. juxtacrine signaling) indicated cocultures as a better representation of in vivo cell characteristics (Holt et al. 2010). Despite the limitations of transwell assays concerning direct cell-cell contacts, they offer the possibility to separate interactions of cells and 3D biomaterials typically occurring during the arrival of cells at the site of injury or during the host response to biomaterials. In such an example, FB recruitment as an answer to the application of poly(lactic acid) scaffolds or chitosan scaffolds colonized with $М \Phi$ was shown to be selectively interfered by additional application of mesenchymal stromal cells in the biomaterial scaffolds (Caires et al. 2018). Another straightforward approach is the coculture of a mixture of cells directly on 2D cell culture scaffolds allowing for intense cell-cell interactions, however, without temporal and spatial control of cell contact and cell type composition (Pan et al. 2011; Steinhauser et al. 1998). Recently, an extension of the classical transwell 


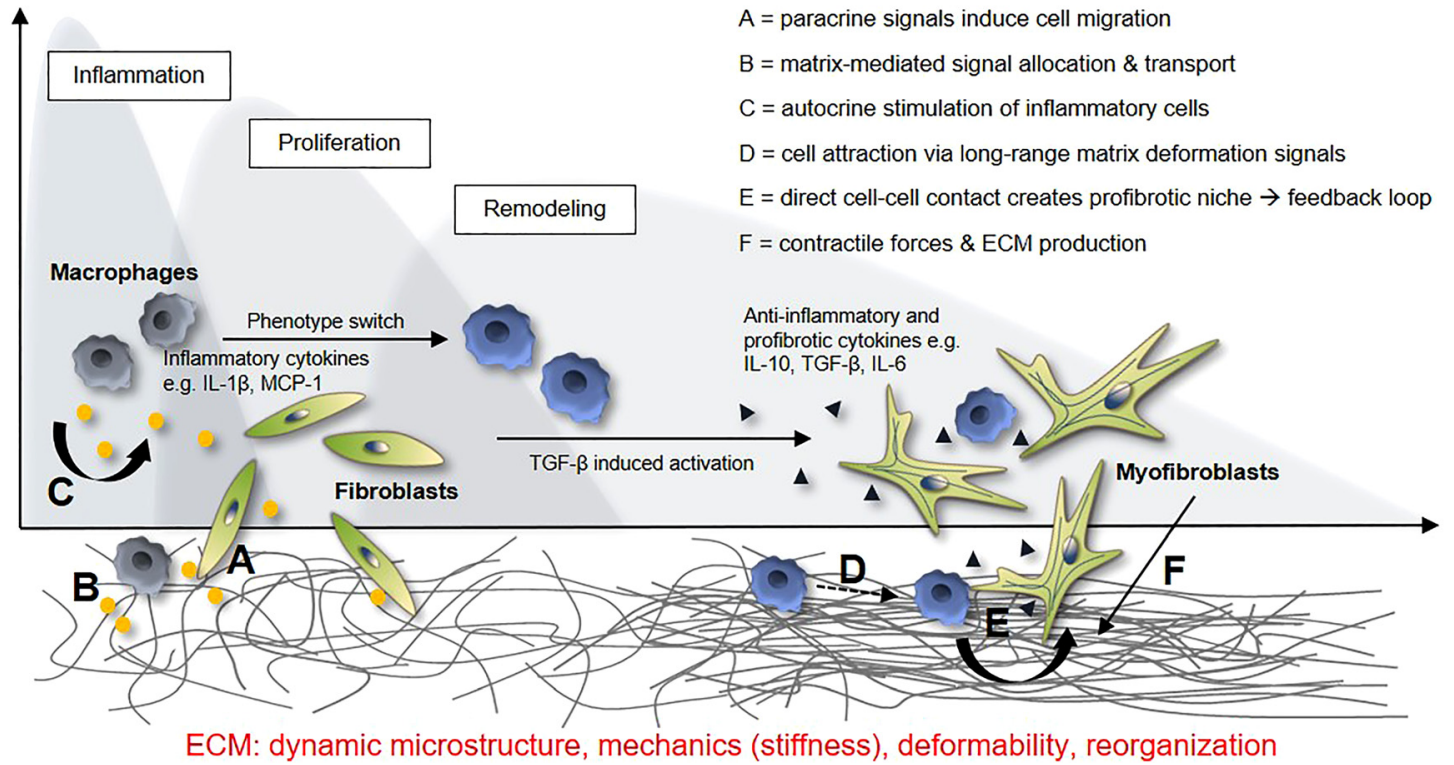

Figure 1: Schematic of the crosstalk between $M \Phi$ and FB during the different stages of wound healing to be addressed in biomimetic models. During inflammation, soluble signals in the wound area induce migration of FB into the early provisional matrix (A) which is facilitated by signal allocation and transport via binding and release at the ECM (B). Besides paracrine signaling, cells are also induced via autocrine signaling (C). With ongoing wound healing, FB become activated by TGF- $\beta$ and mechanical stress transmitted via the ECM and differentiate into MyoFB. MyoFB exerts high contractile forces, resulting in ECM remodeling which also contributes to the attraction of profibrotic M $\Phi$. The juxtacrine vicinity of MyoFB and $M \Phi$ results in the formation of a locally restricted profibrotic niche, thus contributing to the fibrotic cycle (D-F). In the wound resolution phase MyoFB de- or transdifferentiate into other FB cell types supported by paracrine signals like IL-10 (G).

assay with a cell migration fence chamber allowed cells to exchange soluble signals and form homogeneous cell layers at the beginning (Zhou et al. 2015). After fence removal, cells were permitted to migrate and interact to analyze MФ-FB crosstalk with and without juxtacrine interaction in a time-resolved manner. Increased $\mathrm{FB}$ motility and movement of $\mathrm{M \Phi}$ in coculture were observed in comparison to $\mathrm{M} \Phi$ monocultures. Furthermore, $M \Phi$ were shown to promote fibrotic responses by the increase of FB outgrowth as well as EDA-FN and $\alpha$ SMA expression (Zhou et al. 2016).

While the latter model enabled the investigation of autocrine, juxtacrine, and paracrine signals, it lacks a biomimetic 3D context to better mimic the complex parameter space of the wound ECM. This includes microstructure, composition, mechanics, presentation of growth factors and ECM degradation and remodeling which have to be applied to better understand $\mathrm{M} \Phi$-FB crosstalk in early and late stages of wound healing.

\section{In vitro models of early wound healing stages}

The MФ-FB crosstalk in early wound healing has to consider the ECM context of the provisional matrix, which is rich in fibrin, fibronectin and proteoglycans. Various models were engineered to mimic this initial stage, also addressing the foreign body response to implanted biomaterials. Many of the existing models of early wound healing are based on fibrin materials to represent the initial situation in the wound. However, many systems are also based on collagen to mimic the situation in the wound directly after the degradation of the fibrin network and are therefore also of high physiological relevance in the context of early tissue repair. Table 1 provides a comparative overview of the existing coculture models mimicking early and late wound healing stages. A 3D collagen type I scaffold with defects filled with fibrin microparticles was used to coculture human foreskin FB and human epithelial keratinocytes showing an increased keratinocyte migration compared to monoculture conditions (Iyer et al. 2018). In the same constructs a correlation of the high keratinocyte migration with a suppressed proliferation was found for such simulated wound healing. Another model combined blood components of the initial wound, such as primary МФ embedded in a plasma-derived fibrin hydrogel, and studied the exposition of this test system to various biomaterials (glass, titanium, polytetrafluoroethylene [PTFE]) as well as FB integrated in collagen and fibrin matrices (Jannasch et al. 2019). Such a system allowed for in-depth in vitro studies using live cell imaging combined with computational 
modeling addressing the chemotactic potential of the FB and long-term behavior of FB within the 3D collagen matrix. Conditioned media derived from $\mathrm{M} \Phi$ cultured on titanium induced a higher FB motility within the hydrogel, compared to conditioned media derived from МФ cultured on glass or PTFE. The remodeling capacity of FB in terms of collagen deposition and reorganization was significantly higher at PTFE conditions. A coculture model of human foreskin FB and LPS-activated U937 monocyte cell line embedded in a 3D collagen matrix was used to mimic the host inflammatory response (Parks et al. 2014). During a challenge of the coculture system with poly(lactic-co-glycolic acid) (PLGA) particles, increased levels of inflammatory cytokines IL-1 $\beta$, IL-6, GM-CSF and TNF $\alpha$ after 10 days in comparison to monocultures were observed modeling a long-term inflammatory response to the applied biomaterial. Recently, a 2D microfluidic wound-on-a-chip model using two or three cell types (dermal FB, human umbilical vein endothelial cells
[HUVEC], peripheral blood mononuclear cells [PBMC] derived $\mathrm{M} \Phi$ ) was used to study paracrine signals (Biglari et al. 2019). This advanced system made it possible to simulate several essential processes of early wound healing simultaneously: adhesion of cells in a temporary matrix, cell migration from the surrounding tissue and contact with other cells via direct cell-cell communication and via paracrine signals. By that various key steps of wound healing have been modeled including inflammation with increased production of inflammatory cytokines (IL-1ß, IL-6, IL-8), enhanced FB migration and their differentiation into MyoFB, and formation of vascular structures by HUVECs specifically in the case of triple cocultures with M2-МФ. Another recently published model deals with the investigation of МФ-associated functions during the time course of tissue repair by applying collagen matrices with different fibril density in combination with specific MФ subtypes. In detail, the authors (Sapudom et al. 2020) showed that THP-1

Table 1: 3D in vitro coculture models addressing different wound healing stages.

\begin{tabular}{|c|c|c|c|c|}
\hline Design & Scaffold & Cell source & Scope/issue & References \\
\hline \multicolumn{5}{|c|}{ In vitro cocultures associated with early wound healing } \\
\hline Transwell & - & Murine & $\begin{array}{l}\text { Influence of direct versus indirect coculture on cell-cell } \\
\text { signaling }\end{array}$ & Holt et al. (2010) \\
\hline Transwell/direct & - & Human & $\begin{array}{l}\text { FB modulate } M \Phi \text { polarization in coculture towards alterna- } \\
\text { tive activation }\end{array}$ & $\begin{array}{l}\text { Ferrer et al. } \\
(2017)\end{array}$ \\
\hline $\begin{array}{l}\text { Transwell/fence } \\
\text { chamber }\end{array}$ & $\begin{array}{l}\text { Glass with surface } \\
\text { modification }\end{array}$ & Human & MФ stimulate FB outgrowth and promote fibrotic response & $\begin{array}{l}\text { Zhou et al. (2015, } \\
\text { 2016) }\end{array}$ \\
\hline Transwell & $\begin{array}{l}\text { PLA, Chitosan, } \\
\text { Matrigel }\end{array}$ & Human & $\begin{array}{l}\text { Dynamics of cellular recruitment, } \\
M \Phi \text { recruit FB selectively }\end{array}$ & $\begin{array}{l}\text { Caires et al. } \\
(2018)\end{array}$ \\
\hline 3D & $\begin{array}{l}\text { Collagen filled Fibrin } \\
\text { defects }\end{array}$ & Human & Keratinocyte migration increased in presence of FB & lyer et al. (2018) \\
\hline 3D & Collagen, Fibrin & Human & $\begin{array}{l}\text { Development of a screening platform to investigate the } \\
\text { contact of biomaterials to blood components }\end{array}$ & $\begin{array}{l}\text { Jannasch et al. } \\
\text { (2019) }\end{array}$ \\
\hline 3D & Dextran/PLGA & Murine & Prolonged $M \Phi$ activation in direct coculture with $\mathrm{FB}$ & Pan et al. (2011) \\
\hline 3D & $\begin{array}{l}\text { Collagen incl. PLGA } \\
\text { particles }\end{array}$ & Human & $\begin{array}{l}\text { Long-term inflammatory response to PLGA particles in } \\
\text { coculture }\end{array}$ & Parks et al. (2014) \\
\hline 3D & Collagen & Murine & $\begin{array}{l}\text { MФ migration along FB generated far range deformation } \\
\text { fields }\end{array}$ & $\begin{array}{l}\text { Pakshir et al. } \\
\text { (2019) }\end{array}$ \\
\hline \multicolumn{5}{|c|}{ In vitro cocultures associated with late wound healing } \\
\hline Transwell & - & Human & $\begin{array}{l}\text { Increased IL- } 6 \text { release in coculture caused by } M \Phi \\
\text { accumulation }\end{array}$ & Lind et al. (1998) \\
\hline Transwell & - & $\begin{array}{l}\text { Murine, human, } \\
\text { in vivo }\end{array}$ & $\begin{array}{l}\text { Locally restricted activation of latent TGF- } \beta \text { between FB and } \\
M \Phi \text { via Cad11 creates a profibrotic niche }\end{array}$ & $\begin{array}{l}\text { Lodyga et al. } \\
\text { (2019) }\end{array}$ \\
\hline 3D & Collagen & Human & $\begin{array}{l}\text { MФ increase MyoFB activity \& local ECM remodeling in direct } \\
\text { coculture }\end{array}$ & $\begin{array}{l}\text { Mewhort et al. } \\
(2016)\end{array}$ \\
\hline 3D & Collagen & Human & $\begin{array}{l}\text { Augmented collagen contraction by FB in prolonged } \\
\text { coculture }\end{array}$ & Zhu et al. (2001) \\
\hline 3D & Collagen & Murine & $M \Phi$ migration via tunnels or aligned fibers provided by FB & Ford et al. (2019) \\
\hline 3D & Collagen & Human & $\begin{array}{l}\text { M2a-like MФ support the differentiation of FB into MyoFB } \\
\text { depending on collagen fibril density }\end{array}$ & $\begin{array}{l}\text { Sapudom et al. } \\
(2020)\end{array}$ \\
\hline 3D & Collagen & Human & $\begin{array}{l}\text { MФ mediates MyoFB dedifferentiation during late wound } \\
\text { healing stages via secretion of IL-10 }\end{array}$ & Ullm et al. (2020) \\
\hline
\end{tabular}


derived M2a-like MФ are able to sense collagen fibril density. Here, an increased production of TGF- $\beta$ was found in loose collagen matrices, whereas dense matrices suppress TGF- $\beta$ secretion. In direct coculture studies in loose collagen matrices, M2a-like MФ were shown to support the differentiation of FB into MyoFB, which then express higher levels of $\alpha \mathrm{SMA}$, Coll I and EDA-FN. This approach nicely demonstrates how the constitution of the early wound ECM impacts on differentiation and cytokine production of the cells present.

Besides models focusing on the inflammatory response and juxtacrine and paracrine signals, there are some models asking on the induction of cellular infiltration of the wound by $\mathrm{M} \Phi$ and FB. Using a comprehensive analytical toolbox (atomic force microscopy [AFM], confocal laser scanning, time lapse and fluorescence microscopy, cytokine secretion and activity assays) (Ford et al. 2019) investigated collagen fiber alignment, cellular displacement, changes in physical properties of collagen networks by remodeling, MMP activity, and collagen internalization using two cell lines, namely RAW 264.7 MФ and Balb/c 3T3 FB, in 3D collagen matrices of different densities. Collagen fiber alignment by FB in open networks, MMP-dependent matrix degradation and tunnel formation by FB including chemotactic TGF- $\beta$ secretion by FB in dense networks were found to stimulate an increased $\mathrm{M} \Phi$ migration. Another study showed a similar stimulation of MФ migration along FB formed tracks using activated lung FB from mice (with MyoFB phenotype) together with bone marrow $\mathrm{M} \Phi$ from mice tibia and femur (Pakshir et al. 2019). Particle Image Velocimetry (PIV) of deformation fields of the 3D collagen networks generated by contracting MyoFB indicated far-reaching dynamic deformations $(>1300 \mu \mathrm{m})$ by MyoFB. MФ were able to mechanosense these local and dynamic displacements of the collagen network clearly exceeding the range of chemotactic signals (up to $100 \mu \mathrm{m}$ ). Furthermore, the authors demonstrated that the presence of a dynamic force source is critical to initiate directed $M \Phi$ migration, as purely static deformation of the collagen network microstructure were insufficient to stimulate it.

In our group we have also contributed to relevant models of early wound healing. 3D fibrillar collagen networks with well-defined microstructure, composition and elasticity were introduced to mimic various physiological and pathological conditions (Figure 2A) (Franke et al. 2014; Friedemann et al. 2017; Sapudom et al. 2015a, 2017a). In particular, a subsequent modification of such 3D collagen scaffolds with defined amounts of fibronectin allowed to trigger invasion and proliferation of human dermal FB (Figure 2B) (Sapudom et al. 2015b). Differential response of FB and TGF- $\beta$ activated MyoFB in terms of invasion and proliferation have been demonstrated, clearly showing the suitability of such scaffolds to mimic early stages of wound healing in terms of FB infiltration and MyoFB differentiation. In an extension of this model, TGF- $\beta$ releasing microparticles were incorporated in such 3D scaffolds to simulate long-term paracrine signals of other cell types and demonstrated the beneficial application of localized point sources with a slow release of TGF- $\beta$ at physiological levels in the pM range (Figure $2 \mathrm{C}$ ) (Ansorge et al. 2017).
A 3D collagen I scaffold

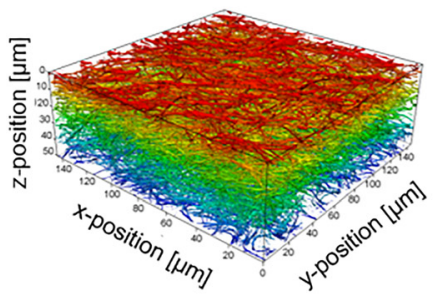

B Fibronectin functionalization

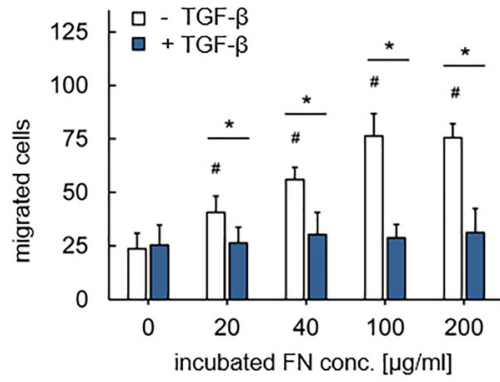

\section{C} Fibroblast differentiation

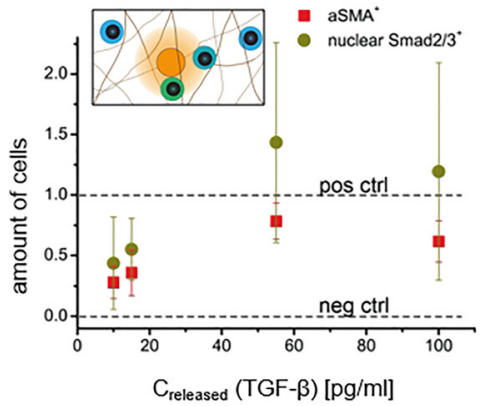

Figure 2: Suitability of collagen-based 3D scaffolds as in vitro models mimicking early wound healing stages. (A) 3D visualization of fibrillar 3D collagen I-based matrices with well-defined microstructure, composition and elasticity. (B) Cell infiltration of primary human foreskin FB into 3D collagen I matrices modified with fibronectin is increased with increasing amounts of bound fibronectin, but is constantly low for TGF- $\beta$ activated MyoFB. Image adapted from Sapudom et al. (2015b). (C) Differentiation of FB into MyoFB have been achieved by the application of TGF- $\beta$ even in the low pM range by slow release from microparticles (schematic illustration in inset), which have been confirmed by the amount of $\alpha$ SMA- and nuclear Smad2/3-positive cells. Image adapted from Ansorge et al. (2017). 


\section{In vitro models of late wound repair}

With progressive wound healing a broad range of cytokines released from multiple cell types act in the inflammatory microenvironment, also initiating a transition to the late stage of wound healing - the remodeling phase. Many processes are intertwined and there are no longer clear transitions between the specific phases, combined by a smooth transition of phenotypes of involved cells including $\mathrm{M} \Phi$ and FB. This situation complicates a better understanding of wound healing and possible pathological outcomes and requires more and better studies including biomimetic in vitro models of late wound repair.

In an early study of FB activation in coculture with $M \Phi$, MФ were challenged with poly(methyl methacrylate) (PMMA) particles in a non-contact coculture using tissue culture inserts and FB activation was measured by the inflammatory mediators released (Lind et al. 1998). The results suggested that the elevated IL- 6 levels found in fibrous tissue may be due to $M \Phi$ accumulation and not primarily caused by particle activation of $M \Phi$. Recently, the activity of human cardiac MyoFB in coculture with human blood monocytes in a collagen-based 3D model was analyzed (Mewhort et al. 2016). A direct contact of MyoFB and monocytes was shown to be essential for collagen gel contraction, local ECM remodeling and MMP9 and TGF- $\beta$ production, in contrast to indirect paracrine cell signals in a transwell system. This observation was confirmed in human and mouse fibrotic lung tissue with emphasizing cell-cell contacts via cadherin11 (Cad11) to be essential to promote the formation of a profibrotic niche (Lodyga et al. 2019). The importance of juxtacrine signals were further supported in in vitro cocultures showing a persistent MyoFB activation by a locally restricted activation of latent TGF- $\beta$ from MyoFB-M $\Phi$ pairs (Lodyga et al. 2019). These findings suggested that the direct contact of $\mathrm{M} \Phi$ and FB in the wound environment may lead to a locally restricted activation of profibrotic processes, which in turn may be a possible cause of fibrosis. Recently, the still unknown influence of M2-MФ-derived exosomes on FB activation was investigated in a coculture of human skin derived FB with THP-1 derived MФ (Chen et al. 2019). In contrast to previous studies focusing on profibrotic factors such as TGF- $\beta$, the authors showed a new MyoFB inducing effect by long noncoding RNA in terms of the expression of glutaminases and aSMA.

Looking at these results, it quickly becomes clear that the former understanding of late wound healing stages and the development of fibrosis has changed. Previous coculture studies, mostly done in 2D, often referred to secretion profiles of certain $\mathrm{M \Phi} \mathrm{subtypes} \mathrm{and} \mathrm{their} \mathrm{effect} \mathrm{on} \mathrm{FB.} \mathrm{There}$ are conclusive findings on how alternatively activated $M \Phi$ influence the profibrotic activities of FB. FB proliferation is increased in coculture with $\mathrm{M} 2-\mathrm{M} \Phi$, as is collagen production and the secretion of other regulatory proteins such as PDGF-BB and PDGF-AA, too, whereas typical inflammatory markers such as TNF $\alpha$ and MMP7 are reduced (Song et al. 2000; Zhu et al. 2017). These findings support long-term experiments with human blood monocytes and fetal lung FB in collagen networks which showed increased collagen network contraction and augmented collagen production under coculture conditions (Zhu et al. 2001).

Besides the overall good understanding of the transition of the inflammatory phase to remodeling and final resolving of the wound, it is still not fully clear how this process is regulated and how remodeling can be stopped at a certain point especially at pathological in vivo conditions. The fate of MyoFB is controversially discussed with early coculture studies on MyoFB apoptosis suggesting IGF-1 derived from IL-4 treated M2-MФ to protect MyoFB from apoptosis after growth factor deprivation (Wynes et al. 2004). Whether MyoFB undergo apoptosis at the end of wound healing, differentiate back into FB or transdifferentiate into other cell types are currently debated. Cocultures of hypertrophic scar FB (HSFs) with human adipose derived stem cells showed, that an initial TGF- $\beta$ mediated increase in the production of collagen I, collagen III, fibronectin, $\alpha$ SMA, IL- 6 , IL-8, and TGF- $\beta$ is remarkably reduced after five days of coculture in a transwell system (Deng et al. 2018). Proliferation, migration and contractility of HSFs was also found to be inhibited. The current discussion was further stimulated by in vivo investigations in mice showing MyoFB to undergo a transdifferentiation into adipocytes (Plikus et al. 2017). Furthermore, it is currently also being investigated to what extent $\mathrm{M} \Phi$ can perceive altered ECM properties in late wound healing and convert them into migratory signals and changes in secretion profile, which in turn affects MyoFB fate (Pakshir et al. 2019).

1ptIn this context our group has considerably contributed to the understanding of MyoFB fate under the influence of paracrine signals of $\mathrm{M} \Phi$, focusing on the soluble mediator IL-10. IL-10 is an anti-inflammatory cytokine that is typically secreted by anti-inflammatory $M \Phi$ during wound resolution and has promising functions in therapy of inflammatory reactions as well as in prevention and reversal of fibrotic processes (Shamskhou et al. 2019; Shi et al. 2013; Wynn and Vannella 2016; Wynn et al. 2013). In our established fibrillar 3D collagen model (see also Figure 2A), the administration of IL-10 to activated MyoFB 
showed that MyoFB do not undergo apoptosis, but revert into an FB phenotype (Sapudom et al. 2017b). The analysis of typical MyoFB markers showed that IL-10 treated MyoFB exhibited lower expression of $\alpha \mathrm{SMA}$, collagen I, collagen III, and EDA-FN, a clear signature of a classical FB phenotype and a transition into a wound resolution phase. In a recently developed coculture system of primary human cells, namely monocyte-derived anti-inflammatory $M \Phi$ and MyoFB derived from human foreskin, IL-10 secretion and expression of MyoFB marker genes in a 3D fibrillar collagen I matrix was investigated (Figure 3A) (Ullm et al. 2020). After six days of coculture of different ratios of $M \Phi$ to MyoFB (1:1 to 5:1) inside the 3D scaffold we observed a ratio-dependent effect on IL-10 cytokine secretion and MyoFB marker gene expression. With increasing amount of anti-inflammatory $M \Phi$, the relative expression of $\alpha S M A$, collagen I, collagen III and EDA-FN was decreased, and we observed a lower inducibility of MyoFB by TGF- $\beta$, clearly indicating a $\mathrm{MФ} \mathrm{controlled} \mathrm{transition} \mathrm{of} \mathrm{MyoFB} \mathrm{into} \mathrm{an} \mathrm{FB}$ phenotype. This effect correlated with an increasing level of IL-10 in the cell culture supernatant with increasing amount of $M \Phi$, identifying the anti-inflammatory $M \Phi$ as the source of IL-10 in the 3D coculture system. The previously suspected effect of IL-10, which counteracts TGF- $\beta$-driven MyoFB activation, was verified hereby. In addition, IL-10 release from cocultured anti-inflammatory $\mathrm{M} \Phi$ was identified as a significant paracrine signal in the coculture system. Thus, our study not only provided a relevant biomimetic 3D ECM-based coculture system for late wound repair, namely wound resolution phase, but it also contributed to a deeper understanding of relevant paracrine signals including IL-10 in MФ-FB crosstalk. In a recent study, we also demonstrated the advantage of such 3D biomimetic systems for in-depth in vitro studies. We showed the time-resolved functional analysis of MyoFB differentiation and dedifferentiation by IL-10 signals using PIV-based and machine-learning assisted contraction studies on the long-term behavior of non-labelled primary FB and MyoFB (Figure 3B) (Riedl and Pompe 2021).

\section{Concluding remarks and outlook}

MФ and FB are crucial players during all phases of wound healing, but the mechanisms of their intercellular communication have not been fully elucidated. It is clear, that the molecular and physical properties of the ECM regulate the behavior of resident cells and modulate the transmission of soluble and mechanical signals. In this review, we have discussed fibrillar 3D matrices and more specifically collagen I based networks as useful scaffolds to study МФ-FB crosstalk in different wound healing stages. In early approaches, in vitro cell studies focused on the application of one cell type and it's response to a huge diversity of newly developed materials, surface modifications, artificial matrices and soluble signals. This rather simplistic approach is increasingly being replaced by using more complex models of cocultures and advanced 3D scaffolds, as these models better allow to study the complex interplay of juxtacrine, paracrine and autocrine signals and the defined adjustment of regulating cues of the ECM microenvironment. Figure 4 provides an overview of
A

MyoFB dedifferentiation in coculture

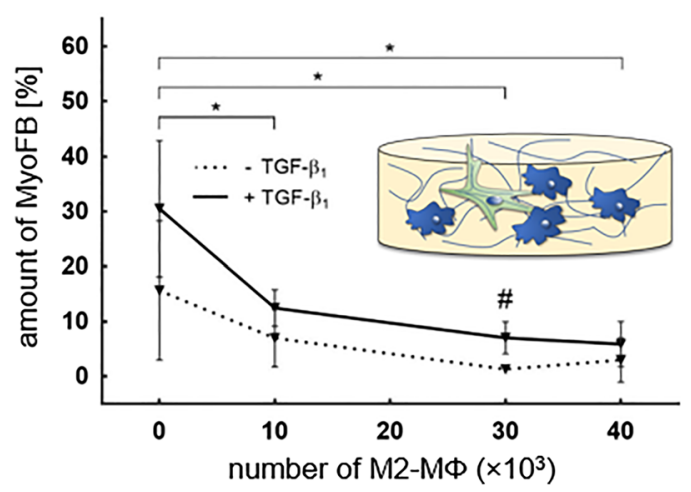

B
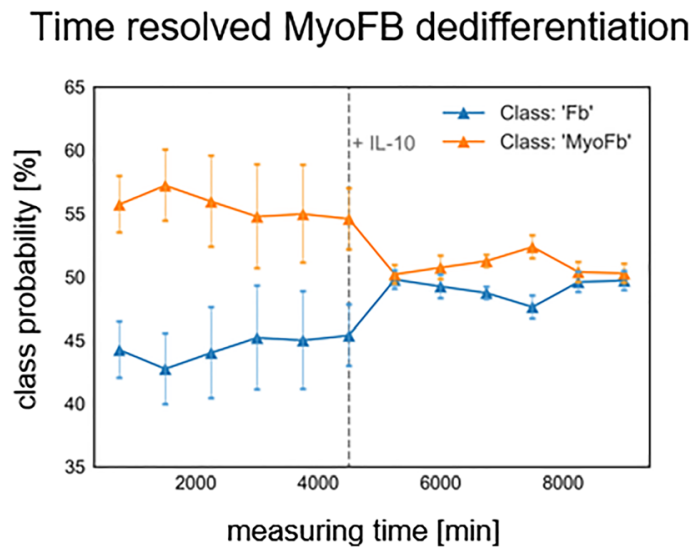

Figure 3: Late stages of wound repair represented by fibrillar 3D collagen-based models.

(A) The inset illustrates the recently developed coculture system of primary human cells, namely monocyte-derived anti-inflammatory $M \Phi$ and MyoFB derived from human foreskin. MyoFB dedifferentiation in 3D collagen matrices have been achieved in coculture in a ratio-dependent manner independent of TGF- $\beta$ presence. Image adapted from Ullm et al. (2020). (B) MyoFB dedifferentiation in 3D collagen matrices can be initiated in monoculture by the addition of a paracrine IL-10 signal. PIV analysis of fibrillar matrix contraction and machine-learning assisted classification allows to follow this process in a time-resolved and label-free manner. Image adapted from Riedl and Pompe (2021). 
the basic principles of in vitro models allowing to study cellular crosstalk during wound repair.

In perspective to get closer to the in vivo state, additional options to modify fibrillar collagen-based scaffolds with other important ECM components, such as GAG, are on the way to be used in wound healing studies. GAG modifications allow to modulate specific interactions of growth factors and cytokines with the surrounding matrix and the cells, including binding, release and presentation, and were already demonstrated to possess antiinflammatory properties (Lohmann et al. 2017; van der Smissen et al. 2013; Zhou and Groth 2018). The control of paracrine and autocrine signals in $\mathrm{M} \Phi$-FB crosstalk by GAG-modified fibrillar 3D matrices is currently addressed in ongoing studies (Figure 5A). Preliminary results of our group show that heparin-modified fibrillar 3D collagen matrices allow to control short-range paracrine signals including chemotactic signals like MCP-1, as studied using microparticle-based release systems and time-resolved single molecules investigations (Figure 5B) (Ansorge et al. 2017).
Further extensions of fibrillar 3D scaffolds for coculture studies include modification of collagen matrices with platelet-rich plasma (do Amaral et al. 2019), and elaborated approaches using artificial skin constructs and animalderived skin. Progress in other research areas using cocultures of MФ and FB such as tumor therapy can also contribute to a better understanding of wound healing, as tumors are frequently discussed as an inflammatory wound, which does not heal. For instance, cancerassociated FB were shown to affect the polarization of tumor-associated $\mathrm{M \Phi}$ towards a protumoral phenotype suppressing the immune response (Hashimoto et al. 2016; Kuen et al. 2017; Takahashi et al. 2017; Zhang et al. 2017).

Besides good progress and perspective in 3D coculture models for wound healing, there are still open issues that should be addressed with future in vitro models. A growing prerequisite for a successful application of new models is the applicability in high-throughput studies associated with reproducibility of model production and defined engineering of specific physiological and pathological conditions. Only in this way, we will be able to predict specific

\section{In vitro cellular crosstalk models of wound repair}

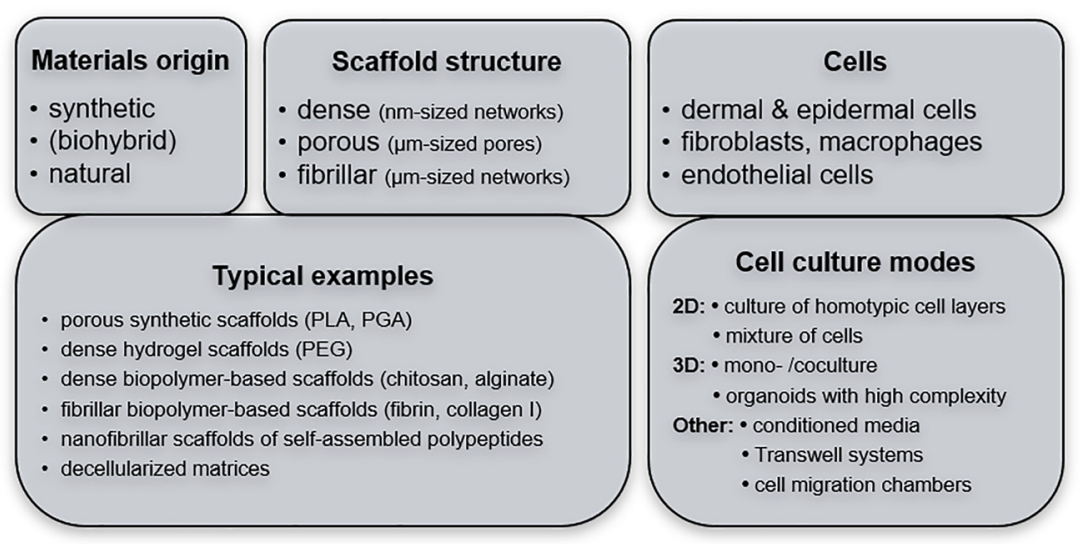

(1)

\section{Mimics of mechanical, biochemical \& structural tissue characteristics}

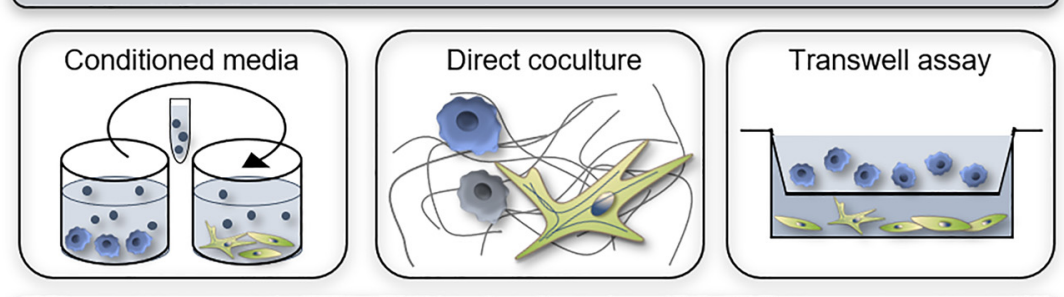

Perspective

mediator gradients, high-throughput screening, single cell tracking
Figure 4: In vitro models of cellular crosstalk in wound repair.

Overview of the basic principles for the design of in vitro models to study cellular crosstalk during wound healing. Typically, existing models in the field of wound healing studies differ between the type of material, scaffold structure, and cell type evaluated. The choice of the type of cell culture (2D, 3D or other) decisively determines the possibilities of interactions to be observed. The common goal of all models is to mimic specific mechanical, biochemical and structural tissue characteristics as closely as possible. Widely used models in the field of cellular interaction studies between macrophages and fibroblasts are based on experiments with conditioned medium, Transwell assays and direct coculture in 3D materials. In perspective, in vitro coculture models are designed to integrate more complex parameters in a defined manner, like mediator gradients, to facilitate reproducible engineering of highthroughput systems and to allow for in-depth analysis of single cell characteristics with high spatial and temporal resolution. 
A

Matrix-modulated MФ-FB crosstalk

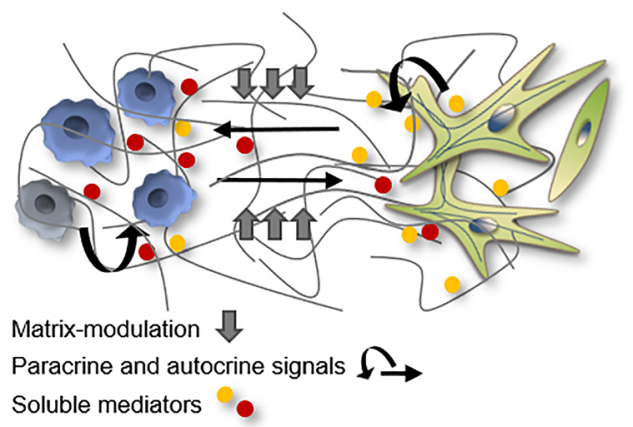

B

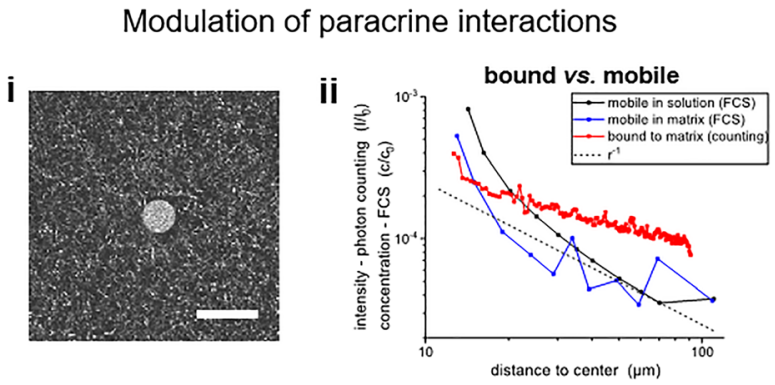

Figure 5: Matrix-modulated paracrine interactions.

(A) Schematic illustration of paracrine and autocrine interactions between MФ and FB modulated by ECM properties. (B) Preliminary results demonstrate, that heparin bound to fibrillar collagen matrices alters the formation and range of mediator gradients (such as MCP-1, TGF- $\beta$ ) released from microparticles as mimics of cellular paracrine signals. (i) Confocal laser scanning image (cLSM) (reflection mode) of a protein loaded microparticle inside a heparin modified fibrillar 3D collagen matrix (scale bar: $40 \mu \mathrm{m}$ ). (ii) Quantified short-range gradients of soluble mediators (here with lysozyme as model protein) in the vicinity of microparticles. cLSM with single molecule sensitivity allowed to measure matrix-bound protein and freely diffusing, mobile protein using photon counting intensity measurements and single point fluorescence correlation spectroscopy (FCS). The heparin modified collagen matrix leads to faster decay of protein concentration for mobile protein (blue line) in comparison to the situation in solution without a matrix environment (black line). The latter concentration gradient nearly follows the theoretically predicted $r^{-1}$ scaling for radial diffusion from a point source (dotted line). Additionally, protein bound to the heparin modified matrix indicates more long-ranged gradients (red line) with slower decay in comparison to free diffusion from a point source (unpublished data, Master thesis of Alexandra Pröger, Leipzig University).

cellular phenotypes and cellular processes that may occur in response to the use of certain materials or therapies (wound matrices, ceramics, drug-releasing micro-particles, other scaffold types). This goes in hand with options to use cells as diagnostic tools to evaluate pathological situations by the occurrence of hybrid МФ phenotypes or phenotype dynamics (Braga et al. 2015).

Author contributions: F.U. and T.P. wrote the manuscript. Research funding: The authors acknowledge the support from Deutsche Forschungsgemeinschaft (DFG, grant: SFB-TRR67/B10). The access to the BioImaging Core Facility (Faculty of Life Sciences, University of Leipzig) supported by a grant from DFG (INST 268/394-1 FUGG) to Tilo Pompe is gratefully acknowledged.

Conflict of interest statement: The authors declare no conflicts of interest regarding this article.

\section{References}

Achterberg, V.F., Buscemi, L., Diekmann, H., Smith-Clerc, J., Schwengler, H., Meister, J.-J., Wenck, H., Gallinat, S., and Hinz, B. (2014). The nano-scale mechanical properties of the extracellular matrix regulate dermal fibroblast function. J. Invest. Dermatol. 134: 1862-1872.

Ansorge, M., Sapudom, J., Chkolnikov, M., Wilde, M., Anderegg, U., Möller, S., Schnabelrauch, M., and Pompe, T. (2017). Mimicking paracrine TGF $\beta 1$ signals during myofibroblast differentiation in 3D collagen networks. Sci. Rep. 7: 5664.

Baker, B.M. and Chen, C.S. (2012). Deconstructing the third dimension: how 3D culture microenvironments alter cellular cues. J. Cell Sci. 125: 3015-3024.

Baker, B.M., Trappmann, B., Wang, W.Y., Sakar, M.S., Kim, I.L., Shenoy, V.B., Burdick, J.A., and Chen, C.S. (2015). Cell-mediated fibre recruitment drives extracellular matrix mechanosensing in engineered fibrillar microenvironments. Nat. Mater. 14: 1262.

Barker, T.H. and Engler, A.J. (2017). The provisional matrix: setting the stage for tissue repair outcomes. Matrix Biol. 60-61: 1-4.

Barron, L. and Wynn, T.A. (2011). Fibrosis is regulated by Th 2 and Th17 responses and by dynamic interactions between fibroblasts and macrophages. Am. J. Physiol. Gastrointest. Liver Physiol. 300: G723-G728.

Biglari, S., Le, T.Y.L., Tan, R.P., Wise, S.G., Zambon, A., Codolo, G., de Bernard, M., Warkiani, M., Schindeler, A., Naficy, S., et al. (2019). Simulating inflammation in a wound microenvironment using a dermal wound-on-a-chip model. Adv. Healthc. Mater. 8: e1801307.

Bonnans, C., Chou, J., and Werb, Z. (2014). Remodelling the extracellular matrix in development and disease. Nat. Rev. Mol. Cell Biol. 15: 786-801.

Braga, T.T., Agudelo, J.S.H., and Camara, N.O.S. (2015). Macrophages during the fibrotic process: M2 as friend and foe. Front. Immunol. 6: 602 .

Bryant, S.J. and Anseth, K.S. (2002). Hydrogel properties influence ECM production by chondrocytes photoencapsulated in poly(ethylene glycol) hydrogels. J. Biomed. Mater. Res. 59: 63-72.

Caires, H.R., Barros da Silva, P., Barbosa, M.A., and Almeida, C.R. (2018). A co-culture system with three different primary human 
cell populations reveals that biomaterials and MSC modulate macrophage-driven fibroblast recruitment. J. Tissue Eng. Regen. Med. 12: e1433-e1440.

Caliari, S.R. and Burdick, J.A. (2016). A practical guide to hydrogels for cell culture. Nat. Methods 13: 405-414.

Chantre, C.O., Hoerstrup, S.P., and Parker, K.K. (2019). Engineering biomimetic and instructive materials for wound healing and regeneration. Curr. Opin. Biomed. Eng. 10: 97-106.

Charras, G. and Sahai, E. (2014). Physical influences of the extracellular environment on cell migration. Nat. Rev. Mol. Cell Biol. 15: 813

Chaudhuri, O., Cooper-White, J., Janmey, P.A., Mooney, D.J., and Shenoy, V.B. (2020). Effects of extracellular matrix viscoelasticity on cellular behaviour. Nature 584: 535-546.

Chen, J., Zhou, R., Liang, Y., Fu, X., Wang, D., and Wang, C. (2019). Blockade of IncRNA-ASLNCS5088-enriched exosome generation in M2 macrophages by GW4869 dampens the effect of M2 macrophages on orchestrating fibroblast activation. FASEB J. Off. Publ. Fed. Am. Soc. Exp. Biol. 33: 12200-12212.

Chouhan, D., Dey, N., Bhardwaj, N., and Mandal, B.B. (2019). Emerging and innovative approaches for wound healing and skin regeneration: current status and advances. Biomaterials 216: 119267.

Damanik, F.F.R., Rothuizen, T.C., van Blitterswijk, C., Rotmans, J.I., and Moroni, L. (2014). Towards an in vitro model mimicking the foreign body response: tailoring the surface properties of biomaterials to modulate extracellular matrix. Sci. Rep. 4: 6325.

Deng, J., Shi, Y., Gao, Z., Zhang, W., Wu, X., Cao, W., and Liu, W. (2018). Inhibition of pathological phenotype of hypertrophic scar fibroblasts via coculture with adipose-derived stem cells. Tissue Eng. 24: 382-393.

Diegelmann, R.F. and Evans, M.C. (2004). Wound healing: an overview of acute, fibrotic and delayed healing. Front. Biosci. 9: 283-289.

do Amaral, R.J.F.C., Zayed, N.M.A., Pascu, E.I., Cavanagh, B., Hobbs, C., Santarella, F., Simpson, C.R., Murphy, C.M., Sridharan, R., González-Vázquez, A., et al. (2019). Functionalising collagenbased scaffolds with platelet-rich plasma for enhanced skin wound healing potential. Front. Bioeng. Biotechnol. 7: 371.

Doyle, A.D. and Yamada, K.M. (2016). Mechanosensing via cell-matrix adhesions in 3D microenvironments. Exp. Cell Res. 343: 60-66.

Edwards, J.P., Zhang, X., Frauwirth, K.A., and Mosser, D.M. (2006). Biochemical and functional characterization of three activated macrophage populations. J. Leukoc. Biol. 80: 1298-1307.

Eming, S.A., Martin, P., and Tomic-Canic, M. (2014). Wound repair and regeneration: mechanisms, signaling, and translation. Sci. Transl. Med. 6: 265sr6.

Eming, S.A., Wynn, T.A., and Martin, P. (2017). Inflammation and metabolism in tissue repair and regeneration. Science 356: 1026-1030.

Fernando, M.R., Giembycz, M.A., and McKay, D.M. (2016) Bidirectional crosstalk via IL-6, PGE2 and PGD2 between murine myofibroblasts and alternatively activated macrophages enhances anti-inflammatory phenotype in both cells. $\mathrm{Br}$. J. Pharmacol. 173: 899-912.

Ferrer, R.A., Saalbach, A., Grünwedel, M., Lohmann, N., Forstreuter, I., Saupe, S., Wandel, E., Simon, J.C., and Franz, S. (2017). Dermal fibroblasts promote alternative macrophage activation improving impaired wound healing. J. Invest. Dermatol. 137: 941-950.
Ford, A.J., Orbach, S.M., and Rajagopalan, P. (2019). Fibroblasts stimulate macrophage migration in interconnected extracellular matrices through tunnel formation and fiber alignment. Biomaterials 209: 88-102.

Franke, K., Sapudom, J., Kalbitzer, L., Anderegg, U., and Pompe, T. (2014). Topologically defined composites of collagen types I and $\mathrm{V}$ as in vitro cell culture scaffolds. Acta Biomater. 10: 2693-2702.

Freudenberg, U., Liang, Y., Kiick, K.L., and Werner, C. (2016). Glycosaminoglycan-based biohybrid hydrogels: a sweet and smart choice for multifunctional biomaterials. Adv. Mater. 28: 8861-8891.

Friedemann, M., Kalbitzer, L., Franz, S., Moeller, S., Schnabelrauch, M., Simon, J.-C., Pompe, T., and Franke, K. (2017). Instructing human macrophage polarization by stiffness and glycosaminoglycan functionalization in 3D collagen networks. Adv. Healthc. Mater. 6: 1600967.

Graney, P.L., Lurier, E.B., and Spiller, K.L. (2018). Biomaterials and bioactive factor delivery systems for the control of macrophage activation in regenerative medicine. ACS Biomater. Sci. Eng. 4: 1137-1148.

Groeber, F., Holeiter, M., Hampel, M., Hinderer, S., and SchenkeLayland, K. (2011). Skin tissue engineering-in vivo and in vitro applications. Adv. Drug Deliv. Rev. 63: 352-366.

Gurtner, G.C., Werner, S., Barrandon, Y., and Longaker, M.T. (2008). Wound repair and regeneration. Nature 453: 314-321.

Haldar, S., Sharma, A., Gupta, S., Chauhan, S., Roy, P., and Lahiri, D. (2019). Bioengineered smart trilayer skin tissue substitute for efficient deep wound healing. Mater. Sci. Eng. C 105: 110140.

Harding, K.G., Morris, H.L., and Patel, G.K. (2002). Healing chronic wounds. BMJ 324: 160-163.

Hashimoto, O., Yoshida, M., Koma, Y.-I., Yanai, T., Hasegawa, D., Kosaka, Y., Nishimura, N., and Yokozaki, H. (2016). Collaboration of cancer-associated fibroblasts and tumour-associated macrophages for neuroblastoma development. J. Pathol. 240: 211-223.

Hinz, B. (2007). Formation and function of the myofibroblast during tissue repair. J. Invest. Dermatol. 127: 526-537.

Hinz, B. (2016). Myofibroblasts. Exp. Eye Res. 142: 56-70.

Holt, D.J., Chamberlain, L.M., and Grainger, D.W. (2010). Cell-cell signaling in co-cultures of macrophages and fibroblasts. Biomaterials 31: 9382-9394.

Infanger, D.W., Lynch, M.E., and Fischbach, C. (2013). Engineered culture models for studies of tumor-microenvironment interactions. Annu. Rev. Biomed. Eng. 15: 29-53.

Iyer, K., Chen, Z., Ganapa, T., Wu, B.M., Tawil, B., and Linsley, C.S. (2018). Keratinocyte migration in a three-dimensional in vitro wound healing model Co-cultured with fibroblasts. Tissue Eng. Regen. Med. 15: 721-733.

Jannasch, M., Gaetzner, S., Groeber, F., Weigel, T., Walles, H., Schmitz, T., and Hansmann, J. (2019). An in vitro model mimics the contact of biomaterials to blood components and the reaction of surrounding soft tissue. Acta Biomater. 89: 227-241.

Jannasch, M., Gaetzner, S., Weigel, T., Walles, H., Schmitz, T., and Hansmann, J. (2017). A comparative multi-parametric in vitro model identifies the power of test conditions to predict the fibrotic tendency of a biomaterial. Sci. Rep. 7: 1689.

Kalbitzer, L., Franke, K., Möller, S., Schnabelrauch, M., and Pompe, T. (2015). Glycosaminoglycan functionalization of mechanically and topologically defined collagen I matrices. J. Mater. Chem. B 3: $8902-8910$ 
Kloc, M., Ghobrial, R.M., Wosik, J., Lewicka, A., Lewicki, S., and Kubiak, J.Z. (2019). Macrophage functions in wound healing. J. Tissue Eng. Regen. Med. 13: 99-109.

Koh, T.J. and DiPietro, L.A. (2011). Inflammation and wound healing: the role of the macrophage. Expet Rev. Mol. Med. 13: e23.

Kuen, J., Darowski, D., Kluge, T., and Majety, M. (2017). Pancreatic cancer cell/fibroblast co-culture induces $\mathrm{M} 2$ like macrophages that influence therapeutic response in a 3D model. PLoS ONE 12: e0182039.

Lang, N.R., Skodzek, K., Hurst, S., Mainka, A., Steinwachs, J., Schneider, J., Aifantis, K.E., and Fabry, B. (2015). Biphasic response of cell invasion to matrix stiffness in three-dimensional biopolymer networks. Acta Biomater. 13: 61-67.

Licup, A.J., Münster, S., Sharma, A., Sheinman, M., Jawerth, L.M., Fabry, B., Weitz, D.A., and MacKintosh, F.C. (2015). Stress controls the mechanics of collagen networks. Proc. Natl. Acad. Sci. USA 112: 9573-9578.

Lind, M., Trindade, M.C., Yaszay, B., Goodman, S.B., and Smith, R.L. (1998). Effects of particulate debris on macrophage-dependent fibroblast stimulation in coculture. J. Bone Joint Surg. Br. 80: 924-930.

Lodyga, M., Cambridge, E., Karvonen, H.M., Pakshir, P., Wu, B., Boo, S., Kiebalo, M., Kaarteenaho, R., Glogauer, M., Kapoor, M., et al. (2019). Cadherin-11-mediated adhesion of macrophages to myofibroblasts establishes a profibrotic niche of active TGF- $\beta$. Sci. Signal. 12: eaao3469.

Logie, C., van Schaik, T., Pompe, T., and Pietsch, K. (2020). Fibronectin-functionalization of 3D collagen networks supports immune tolerance and inflammation suppression in human monocyte-derived macrophages. Biomaterials 268: 120498.

Lohmann, N., Schirmer, L., Atallah, P., Wandel, E., Ferrer, R.A., Werner, C., Simon, J.C., Franz, S., and Freudenberg, U. (2017).

Glycosaminoglycan-based hydrogels capture inflammatory chemokines and rescue defective wound healing in mice. Sci. Transl. Med. 9: eaai9044.

Lutolf, M.P. and Hubbell, J.A. (2005). Synthetic biomaterials as instructive extracellular microenvironments for morphogenesis in tissue engineering. Nat. Biotechnol. 23: 47.

Lutolf, M.P., Lauer-Fields, J.L., Schmoekel, H.G., Metters, A.T., Weber, F.E., Fields, G.B., and Hubbell, J.A. (2003). Synthetic matrix metalloproteinase-sensitive hydrogels for the conduction of tissue regeneration: engineering cell-invasion characteristics. Proc. Natl. Acad. Sci. USA 100: 5413-5418.

Mantovani, A., Sica, A., Sozzani, S., Allavena, P., Vecchi, A., and Locati, M. (2004). The chemokine system in diverse forms of macrophage activation and polarization. Trends Immunol. 25: 677-686.

Martin, K.E. and García, A.J. (2021). Macrophage phenotypes in tissue repair and the foreign body response: Implications for biomaterial-based regenerative medicine strategies. Acta Biomater., https://doi.org/10.1016/j.actbio.2021.03.038 (Epub ahead of print).

Martinez, F.O., Sica, A., Mantovani, A., and Locati, M. (2008). Macrophage activation and polarization. Front. Biosci. 13: 453-461.

Metcalfe, A.D. and Ferguson, M.W.J. (2007). Tissue engineering of replacement skin: the crossroads of biomaterials, wound healing, embryonic development, stem cells and regeneration. J. R. Soc. Interface 4: 413-437.
Mewhort, H.E.M., Lipon, B.D., Svystonyuk, D.A., Teng, G., Guzzardi, D.G., Silva, C., Yong, V.W., and Fedak, P.W.M. (2016). Monocytes increase human cardiac myofibroblast-mediated extracellular matrix remodeling through TGF- $\beta 1$. Am. J. Physiol. Heart Circ. Physiol. 310: H716-H724.

Minutti, C.M., Knipper, J.A., Allen, J.E., and Zaiss, D.M.W. (2017). Tissue-specific contribution of macrophages to wound healing. Semin. Cell Dev. Biol. 61: 3-11.

Nathan, C. and Shiloh, M.U. (2000). Reactive oxygen and nitrogen intermediates in the relationship between mammalian hosts and microbial pathogens. Proc. Natl. Acad. Sci. USA 97: 8841-8848.

Oka, T., Ohta, K., Kanazawa, T., and Nakamura, K.-I. (2016). Interaction between macrophages and fibroblasts during wound healing of burn injuries in rats. Kurume Med. J. 62: 59-66.

Pakshir, P., Alizadehgiashi, M., Wong, B., Coelho, N.M., Chen, X., Gong, Z., Shenoy, V.B., McCulloch, C.A., and Hinz, B. (2019). Dynamic fibroblast contractions attract remote macrophages in fibrillar collagen matrix. Nat. Commun. 10: 1850.

Pakshir, P. and Hinz, B. (2018). The big five in fibrosis: macrophages, myofibroblasts, matrix, mechanics, and miscommunication. Matrix Biol. 68: 81-93.

Pan, H., Jiang, H., Kantharia, S., and Chen, W. (2011). A fibroblast/ macrophage co-culture model to evaluate the biocompatibility of an electrospun Dextran/PLGA scaffold and its potential to induce inflammatory responses. Biomed. Mater. 6: 65002.

Parks, A.C., Sung, K., and Wu, B.M. (2014). A three-dimensional in vitro model to quantify inflammatory response to biomaterials. Acta Biomater. 10: 4742-4749.

Patel, U., Rajasingh, S., Samanta, S., Cao, T., Dawn, B., and Rajasingh, J. (2017). Macrophage polarization in response to epigenetic modifiers during infection and inflammation. Drug Discov. Today 22: 186-193.

Place, E.S., Evans, N.D., and Stevens, M.M. (2009). Complexity in biomaterials for tissue engineering. Nat. Mater. 8: 457-470.

Plikus, M.V., Guerrero-Juarez, C.F., Ito, M., Li, Y.R., Dedhia, P.H., Zheng, Y., Shao, M., Gay, D.L., Ramos, R., Hsi, T.-C., et al. (2017). Regeneration of fat cells from myofibroblasts during wound healing. Science 355: 748-752.

Ploeger, D.T., Hosper, N.A., Schipper, M., Koerts, J.A., de Rond, S., and Bank, R.A. (2013). Cell plasticity in wound healing: paracrine factors of M1/M2 polarized macrophages influence the phenotypical state of dermal fibroblasts. Cell Commun. Signal. 11: 29.

Prewitz, M., Seib, F.P., Pompe, T., and Werner, C. (2012). Polymeric biomaterials for stem cell bioengineering. Macromol. Rapid Commun. 33: 1420-1431.

Rahmany, M.B. and van Dyke, M. (2013). Biomimetic approaches to modulate cellular adhesion in biomaterials: a review. Acta Biomater. 9: 5431-5437.

Riedl, P. and Pompe, T. (2021). Functional label-free assessment of fibroblast differentiation in 3D collagen-I-matrices using particle image velocimetry. Biomater. Sci. (accepted), https://doi.org/ 10.1039/D1BM00638J.

Rodero, M.P. and Khosrotehrani, K. (2010). Skin wound healing modulation by macrophages. Int. J. Clin. Exp. Pathol. 3: 643-653.

Rohani, M.G. and Parks, W.C. (2015). Matrix remodeling by MMPs during wound repair. Matrix Biol. 44: 113-121. 
Rowley, A.T., Nagalla, R.R., Wang, S.-W., and Liu, W.F. (2019). Extracellular matrix-based strategies for immunomodulatory biomaterials engineering. Adv. Healthc. Mater. 8: e1801578.

Sadtler, K., Sommerfeld, S.D., Wolf, M.T., Wang, X., Majumdar, S., Chung, L., Kelkar, D.S., Pandey, A., and Elisseeff, J.H. (2017). Proteomic composition and immunomodulatory properties of urinary bladder matrix scaffolds in homeostasis and injury. Semin. Immunol. 29: 14-23.

Saeed, S., Quintin, J., Kerstens, H.H.D., Rao, N.A., Aghajanirefah, A., Matarese, F., Cheng, S.-C., Ratter, J., Berentsen, K., van der Ent, M.A., et al. (2014). Epigenetic programming of monocyte-tomacrophage differentiation and trained innate immunity. Science 345: 1251086.

Sapudom, J., Kalbitzer, L., Wu, X., Martin, S., Kroy, K., and Pompe, T. (2019). Fibril bending stiffness of 3D collagen matrices instructs spreading and clustering of invasive and non-invasive breast cancer cells. Biomaterials 193: 47-57.

Sapudom, J., Mohamed, W.K.E., Garcia-Sabaté, A., Alatoom, A., Karaman, S., Mahtani, N., and Teo, J.C (2020). Collagen fibril density modulates macrophage activation and cellular functions during tissue repair. Bioengineering 7: 33 .

Sapudom, J. and Pompe, T. (2018). Biomimetic tumor microenvironments based on collagen matrices. Biomater. Sci. 6: 2009-2024.

Sapudom, J., Rubner, S., Martin, S., Kurth, T., Riedel, S., Mierke, C.T., and Pompe, T. (2015a). The phenotype of cancer cell invasion controlled by fibril diameter and pore size of 3D collagen networks. Biomaterials 52: 367-375.

Sapudom, J., Rubner, S., Martin, S., Thoenes, S., Anderegg, U., and Pompe, T. (2015b). The interplay of fibronectin functionalization and TGF- $\beta 1$ presence on fibroblast proliferation, differentiation and migration in 3D matrices. Biomater. Sci. 3: 1291-1301.

Sapudom, J., Ullm, F., Martin, S., Kalbitzer, L., Naab, J., Möller, S., Schnabelrauch, M., Anderegg, U., Schmidt, S., and Pompe, T. (2017a). Molecular weight specific impact of soluble and immobilized hyaluronan on CD44 expressing melanoma cells in 3D collagen matrices. Acta Biomater. 50: 259-270.

Sapudom, J., Wu, X., Chkolnikov, M., Ansorge, M., Anderegg, U., and Pompe, T. (2017b). Fibroblast fate regulation by time dependent TGF- $\beta 1$ and IL-10 stimulation in biomimetic 3D matrices. Biomater. Sci. 5: 1858-1867.

Sapudom, J., Wu, X., Chkolnikov, M., Ansorge, M., Anderegg, U., and Pompe, T. (2017c). Fibroblast fate regulation by time dependent TGF- $\beta 1$ and IL-10 stimulation in biomimetic 3D matrices. Biomater. Sci. 5: 1858-1867.

Seo, B.R., Chen, X., Ling, L., Song, Y.H., Shimpi, A.A., Choi, S., Gonzalez, J., Sapudom, J., Wang, K., Andresen Eguiluz, R.C., et al. (2020). Collagen microarchitecture mechanically controls myofibroblast differentiation. PNAS 117: 11387-11398.

Shamskhou, E.A., Kratochvil, M.J., Orcholski, M.E., Nagy, N., Kaber, G., Steen, E., Balaji, S., Yuan, K., Keswani, S., Danielson, B., et al. (2019). Hydrogel-based delivery of Il-10 improves treatment of bleomycin-induced lung fibrosis in mice. Biomaterials 203: $52-62$.

Shi, J.-H., Guan, H., Shi, S., Cai, W.-X., Bai, X.-Z., Hu, X.-L., Fang, X.-B., Liu, J.-Q., Tao, K., Zhu, X.-X., et al. (2013). Protection against TGF$\beta 1$-induced fibrosis effects of IL-10 on dermal fibroblasts and its potential therapeutics for the reduction of skin scarring. Arch. Dermatol. Res. 305: 341-352.

Shook, B.A., Wasko, R.R., Rivera-Gonzalez, G.C., Salazar-Gatzimas, E., López-Giráldez, F., Dash, B.C., Muñoz-Rojas, A.R., Aultman, K.D.,
Zwick, R.K., Lei, V., et al. (2018). Myofibroblast proliferation and heterogeneity are supported by macrophages during skin repair. Science 362: aar2971.

Sindrilaru, A. and Scharffetter-Kochanek, K. (2013). Disclosure of the Culprits: macrophages-versatile regulators of wound healing. Adv. Wound Care 2: 357-368.

Singer, A.J. and Clark, R.A. (1999). Cutaneous wound healing. N. Engl. J. Med. 341: 738-746.

Snyder, R.J., Lantis, J., Kirsner, R.S., Shah, V., Molyneaux, M., and Carter, M.J. (2016). Macrophages: a review of their role in wound healing and their therapeutic use. Wound Repair Regen. 24: 613-629.

Song, E., Ouyang, N., Hörbelt, M., Antus, B., Wang, M., and Exton, M.S. (2000). Influence of alternatively and classically activated macrophages on fibrogenic activities of human fibroblasts. Cell. Immunol. 204: 19-28.

Sottile, J. and Hocking, D.C. (2002). Fibronectin polymerization regulates the composition and stability of extracellular matrix fibrils and cell-matrix adhesions. Mol. Biol. Cell 13: 3546-3559.

Stamov, D.R., Khoa Nguyen, T.A., Evans, H.M., Pfohl, T., Werner, C., and Pompe, T. (2011). The impact of heparin intercalation at specific binding sites in telopeptide-free collagen type I fibrils. Biomaterials 32: 7444-7453.

Steinhauser, M.L., Kunkel, S.L., Hogaboam, C.M., Evanoff, H., Strieter, R.M., and Lukacs, N.W. (1998). Macrophage/fibroblast coculture induces macrophage inflammatory protein-1alpha production mediated by intercellular adhesion molecule- 1 and oxygen radicals. J. Leukoc. Biol. 64: 636-641.

Takahashi, H., Sakakura, K., Kudo, T., Toyoda, M., Kaira, K., Oyama, T., and Chikamatsu, K. (2017). Cancer-associated fibroblasts promote an immunosuppressive microenvironment through the induction and accumulation of protumoral macrophages. Oncotarget 8: 8633-8647.

Ter Horst, B., Chouhan, G., Moiemen, N.S., and Grover, L.M. (2018). Advances in keratinocyte delivery in burn wound care. Adv. Drug Deliv. Rev. 123: 18-32.

Tomasek, J.J., Gabbiani, G., Hinz, B., Chaponnier, C., and Brown, R.A. (2002). Myofibroblasts and mechano-regulation of connective tissue remodelling. Nat. Rev. Mol. Cell Biol. 3: 349-363.

Tsurkan, M.V., Chwalek, K., Levental, K.R., Freudenberg, U., and Werner, C. (2010). Modular StarPEG-heparin gels with bifunctional peptide linkers. Macromol. Rapid Commun. 31: 1529-1533.

Ullm, F., Riedl, P., Machado de Amorim, A., Patzschke, A., Weiß, R., Hauschildt, S., Franke, K., Anderegg, U., and Pompe, T. (2020). 3D scaffold-based macrophage fibroblast coculture model reveals IL-10 dependence of wound resolution phase. Adv. Biosyst. 4: 1900220.

van der Smissen, A., Samsonov, S., Hintze, V., Scharnweber, D., Moeller, S., Schnabelrauch, M., Pisabarro, M.T., and Anderegg, U. (2013). Artificial extracellular matrix composed of collagen I and highly sulfated hyaluronan interferes with TGF $\beta(1)$ signaling and prevents TGF $\beta$ (1)-induced myofibroblast differentiation. Acta Biomater. 9: 7775-7786.

van der Veen, V.C., van der Wal, M.B.A., van Leeuwen, M.C.E., Ulrich, M.M.W., and Middelkoop, E. (2010). Biological background of dermal substitutes. Burns J. Int. Soc. Burn Inj. 36: 305-321.

van Linthout, S., Miteva, K., and Tschöpe, C. (2014). Crosstalk between fibroblasts and inflammatory cells. Cardiovasc. Res. 102: 258-269. 
Venter, C. and Niesler, C. (2018). A triple co-culture method to investigate the effect of macrophages and fibroblasts on myoblast proliferation and migration. BioTechniques 64: 52-58.

Werner, S. and Grose, R. (2003). Regulation of wound healing by growth factors and cytokines. Physiol. Rev. 83: 835-870.

Whelan, D., Caplice, N.M., and Clover, A.J.P. (2014). Fibrin as a delivery system in wound healing tissue engineering applications. J. Contr. Release 196: 1-8.

Witherel, C.E., Graney, P.L., Freytes, D.O., Weingarten, M.S., and Spiller, K.L. (2016). Response of human macrophages to wound matrices in vitro. Wound Repair Regen. 24: 514-524.

Wynes, M.W., Frankel, S.K., and Riches, D.W.H. (2004). IL-4-induced macrophage-derived IGF-I protects myofibroblasts from apoptosis following growth factor withdrawal. J. Leukoc. Biol. 76: 1019-1027.

Wynn, T.A. and Vannella, K.M. (2016). Macrophages in tissue repair, regeneration, and fibrosis. Immunity 44: 450-462.

Wynn, T.A., Chawla, A., and Pollard, J.W. (2013). Macrophage biology in development, homeostasis and disease. Nature 496: 445-455.

Xue, J., Wang, X., Wang, E., Li, T., Chang, J., and Wu, C. (2019). Bioinspired multifunctional biomaterials with hierarchical microstructure for wound dressing. Acta Biomater. 100: 270-279.

Yanez, D.A., Lacher, R.K., Vidyarthi, A., and Colegio, O.R. (2017). The role of macrophages in skin homeostasis. Pflugers Arch. Eur. J. Physiol. 469: 455-463.

Zhou, G. and Groth, T. (2018). Host responses to biomaterials and antiinflammatory design - a brief review. Macromol. Biosci. 18: 1800112.

Zhou, G., Loppnow, H., and Groth, T. (2015). A macrophage/fibroblast co-culture system using a cell migration chamber to study inflammatory effects of biomaterials. Acta Biomater. 26: 54-63.

Zhou, G., Liedmann, A., Chatterjee, C., and Groth, T. (2016). In vitro study of the host responses to model biomaterials via a fibroblast/ macrophage co-culture system. Biomater. Sci. 5: 141-152.

Zhang, A., Qian, Y., Ye, Z., Chen, H., Xie, H., Zhou, L., Shen, Y., and Zheng, S. (2017). Cancer-associated fibroblasts promote M2 polarization of macrophages in pancreatic ductal adenocarcinoma. Canc. Med. 6: 463-470.

Zhu, Y., Sköld, C.M., Liu, X., Wang, H., Kohyama, T., Wen, F.Q., Ertl, R.F., and Rennard, S.I. (2001). Fibroblasts and monocyte macrophages contract and degrade three-dimensional collagen gels in extended co-culture. Respir. Res. 2: 295-299.

Zhu, Z., Ding, J., Ma, Z., Iwashina, T., and Tredget, E.E. (2017). Alternatively activated macrophages derived from THP-1 cells promote the fibrogenic activities of human dermal fibroblasts. Wound Repair Regen. 25: 377-388.

\section{Bionotes}

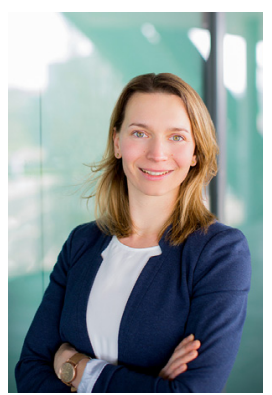

Franziska Ullm

Faculty of Life Sciences, Institute of

Biochemistry, Leipzig University, D-04103

Leipzig, Germany

https://orcid.org/0000-0002-0547-7540

Franziska Ullm is a Ph.D. candidate in Biophysical Chemistry at Universität Leipzig. Her current research, under the advisement of Prof. Tilo Pompe, is focused on the development of a 3D in vitro coculture model of $M \Phi$ and FB based on collagen I. She is interested in the dynamic cell-cell and cell-matrix crosstalk in the context of different wound healing situations. She obtained her master's degree in biochemistry from Universität Leipzig in 2015.

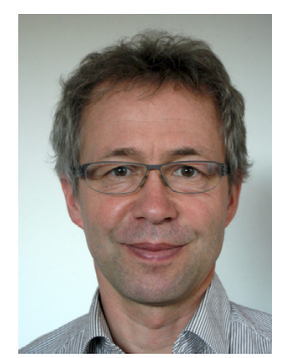

\section{Tilo Pompe}

Faculty of Life Sciences, Institute of Biochemistry, Leipzig University, D-04103 Leipzig, Germany tilo.pompe@uni-leipzig.de https://orcid.org/0000-0003-1508-4959

Tilo Pompe received his PhD from the Max-Planck-Institute for Colloids and Interfaces in Golm-Potsdam in 2000. After being a postdoc and group leader at the Max-Bergmann Center of Biomaterials at the Leibniz Institute of Polymer Research Dresden until 2011, he became a Professor for Biophysical Chemistry at the Institute of Biochemistry at Universität Leipzig. His main research interests are bioengineering of biomimetic materials based on synthetic and naturally derived polymers including usage of these materials to answer biomedical questions on microenvironmental cues in cell fate decisions and to develop new biosensing systems for anthropogenic analytes. 